\title{
Blood Safety: On-Site Verification Of The Screening Of HIV, HBV And HCV By Enzyme Linked Immuno-Sorbent Assay (ELISA) Kits From Bio-Rad Laboratories At The "Centre National De Transfusion Sanguine” (CNTS) Of Lomé In Togo
}

\author{
Gnatoulma Katawa \\ Christèle Nguepou Tchopba \\ Marthe Oukoé Amessoudji \\ Pelagie Edlom Tchadié \\ Simplice Damintoti Karou \\ Yaovi Ameyapoh
}

Ecole Supérieure des Techniques Biologiques et Alimentaires

(ESTBA)/Laboratoire de Microbiologie et de Contrôle de Qualité des

Denrées Alimentaires (LAMICODA)/Unité de Recherche en Immunologie et Immunomodulation (UR2IM), Université de Lomé, Togo

\section{Liza Koboyo Nadjir \\ Ablam Ameyissa \\ Lochina Feteke}

Centre National de Transfusion Sanguine (CNTS) de Lomé, Togo

\section{Esso-Mondjonna Tougoma}

Ecole Supérieure des Techniques Biologiques et Alimentaires

(ESTBA)/Laboratoire de Microbiologie et de Contrôle de Qualité des

Denrées Alimentaires (LAMICODA)/Unité de Recherche en Immunologie et Immunomodulation (UR2IM), Université de Lomé, Togo

Centre National de Transfusion Sanguine (CNTS) de Lomé, Togo

\section{Doi:10.19044/esj.2021.v17n21p92}

Submitted: 22 February 2021

Accepted: 19 May 2021

Published: 30 June 2021
Copyright 2021 Author(s)

Under Creative Commons BY-NC-ND 4.0 OPEN ACCESS

Cite As:

Katawa G., Nguepou Tchopba C., Amessoudji M.O., Tchadié P.E., Karou S.D., Ameyapoh Y., Nadjir L.K., Ameyissa A., Feteke L. \& Tougoma E-M. (2021). Blood Safety: On-Site Verification Of The Screening Of HIV, HBV And HCV By Enzyme Linked Immuno-Sorbent Assay (ELISA) Kits From Bio-Rad Laboratories At The "Centre National De Transfusion Sanguine” (CNTS) Of Lomé In Togo. European Scientific Journal, ESJ, 17(21), 92. https://doi.org/10.19044/esj.2021.v17n21p92 


\section{Abstract}

Background: Blood transfusion improves health and saves lives. Safe blood must be ensured for our populations. Quality assurance is a process that includes a set of coordinated activities in order to achieve the quality objective. Compliance with the quality management rules of medical biology laboratories requires verification of methods prior to their use. This study aimed to verify the on-site verification of the performance of the Enzyme Linked Immuno-Sorbent Assay (ELISA) method performed at the serology laboratory of the CNTS of Lomé.

Methods: The performance of ELISA method performed at the serology laboratory of CNTS for the diagnosis of HIV, Hepatitis B and C with Bio-Rad Genscreen ULTRA-HIV Ag-Ac, Bio-Rad Monolisa-HBs Ag ULTRA and Bio-Rad Monolisa HCV Ag-Ac ULTRA V2 kits respectively was evaluated on repeatability, reproducibility, sensitivity and specificity according to COFRAC's SH GTA 04 reference.

Results: The evaluation of the repeatability and reproducibility of each kit used in the laboratory resulted in compliant Coefficients of Variation (CV) with manufacturers' ones. Sensitivities obtained with Bio-Rad Monolisa HCV Ag-Ac ULTRA V2, Bio-Rad Monolisa HBs Ag ULTRA and Bio-Rad Genscreen ULTRA HIV Ag-Ac kits were 94.59\%, 98.08\% and 100\% respectively. For specificity tests, we found $86.49 \%$ with BIO-Rad Genscreen ULTRA-HIV Ag-Ac kit, 94.34\% with Bio-Rad Monolisa HCV Ag-Ac ULTRA V2 kit and 97.37\% with Bio-Rad Monolisa-HBs Ag ULTRA.

Conclusion: In general, results were compliant except HIV diagnosis specificity. This study appears as a contribution to the establishment of a verification file for ELISA method used at the serology laboratory of CNTS of Lomé.

Keywords: Blood Safety, Quality Assurance, Method Verification, ELISA, Repeatability, Reproducibility, Sensitivity, Specificity

\section{Introduction}

Blood transfusion is a very important health procedure. It is therefore the responsibility of the national blood transfusion program to ensure the quality of blood products for a secure clinical use (WHO, 2010). Quality assurance is a process that includes a set of coordinated activities in order to achieve the quality objective (ISO 9000, 2015). When analyses are performed, there is always some degree of uncertainty. The challenge is to reduce the level of inaccuracy as much as possible, and this can only be achieved through mastering analytical systems and methods (Marques-Garcia et al., 2015). In August 2015, Togo Ministry of Health and Social Protection (MHSP) issued a decree $\mathrm{N}^{\circ} 115 / 2015 / \mathrm{MSPS} / \mathrm{CAB} / \mathrm{SG}$ to institute ISO 15189 standard as a 
reference for all medical biology laboratories in Togo (Cucherat, 2017). In order to meet this challenge outlined above, the standard specifies in its paragraph 5.5.1.3, the requirements for the validation of analytical procedures (COFRAC, 2016). Validation of method is confirmation, through the provision of objective evidence, that the requirements for a specific intended use or application have been fulfilled (International Standard Organization, 2005). Compliance with the quality management rules of medical biology laboratories requires verification of methods prior to their use. The "Centre National de Transfusion Sanguine" (CNTS) is the national reference center for blood transfusion in Togo. The biological qualification of donations is done in two laboratories of CNTS, namely serology and immunohematology laboratories. Both must meet the requirements of the ISO15189 standard adopted by the MHSP. Thus, from April 28 to 29, 2016, a diagnostic audit, commissioned by the Division of Laboratories, was carried out in these two laboratories in regard to the above-mentioned reference system. One of the discrepancies noted during this diagnostic audit was the non-verification of methods and analytical procedures. This study responds to this challenge by verifying ELISA method with kits from Bio-Rad Laboratories used in the serology laboratory of the CNTS in order to ensure safe blood to our population.

\section{Methods}

This was a descriptive study, from January 15 to February 20, 2018 at the serology laboratory of CNTS in Lomé.

Biological samples consisting of HIV, HBV, HCV positive and negative sera were provided by the National Reference Center (NRC) and CNTS serum bank respectively.

The performance of the ELISA method used at the serology laboratory of CNTS for the diagnosis of HIV, Hepatitis B and C with Bio-Rad Genscreen ULTRA-HIV Ag-Ac, Bio-Rad Monolisa-HBs Ag ULTRA and Bio-Rad Monolisa HCV Ag-Ac ULTRA V2 kits respectively, has been evaluated on repeatability, reproducibility, sensitivity and specificity according to COFRAC's SH GTA 04 reference (COFRAC, 2015).

\section{Repeatability assessment}

To assess the repeatability, negative and positive controls of ELISA kits were tested 17 times under the same conditions by the same scientists and coefficients of variation (CV) were calculated. 


\section{Reproducibility assessment}

To evaluate the reproducibility, negative and positive controls of ELISA kits were analyzed 17 times under different conditions by changing scientists and coefficients of variation (CV) were calculated.

$$
\mathrm{CV}=(\mathrm{SD} / \mathrm{m}) * \mathbf{1 0 0}
$$

$\mathbf{S D}=$ Standard deviation; $\mathbf{m}=$ mean

\section{Evaluation of sensitivity and specificity of the methods}

For the assessment of sensitivity and specificity, pools of sera known as positive or negative for either HIV, HBV or HCV were tested. Sensitivity and specificity was determined as follow:

$$
\begin{gathered}
\text { Sensitivity }=\mathbf{T P} /(\mathrm{TP}+\mathrm{FN}) * \mathbf{1 0 0} \\
\text { Specificity }=\mathbf{T N} /(\mathrm{TN}+\mathbf{F P}) * \mathbf{1 0 0}
\end{gathered}
$$

\section{Evaluation of predictive values}

Positive predictive values (PPV) and negative predictive values (NPV) were calculated as follow:

$$
\begin{aligned}
& \mathbf{P P V}=\mathbf{T P} /(\mathbf{T P}+\mathbf{F P}) * 100 \\
& \mathrm{NPV}=\mathrm{TN} /(\mathbf{T N}+\mathbf{F N}) * 100
\end{aligned}
$$

$\mathrm{TP}=$ True positive; $\mathrm{FP}=$ False positive; $\mathrm{FN}=$ False negative; $\mathrm{TN}=$ True negative

\section{Statistical analysis}

Descriptive statistics were performed using Graph Pad PRISM 5.02 software (GraphPad Software, San Diego California USA).

\section{Results \\ Evaluation of repeatability}

Table 1 shows the results of repeatability test of the ELISA method with different kits. It appears that the CV of negative control (5.28\%) and positive control (2.69\%) of BIO-Rad Genscreen ULTRA-HIV Ag-Ac dedicated to the diagnosis of HIV, are lower than the manufacturer's ones which are respectively $5.37 \%$ and $8.48 \%$. For the diagnosis of Hepatitis B, the negative control of Bio-Rad Monolisa-HBs Ag ULTRA gives a CV of 6.83\%, lower than the manufacturer's one (10.6\%) and the positive control gives a CV of $2.38 \%$, also lower than the manufacturer's one (6.2\%). Regarding the diagnosis of Hepatitis C, the trend is the same with Bio-Rad Monolisa HCV Ag-Ac ULTRA V2 which negative control CV (6.38\%) and positive control CV (3.83\%) are also lower than the manufacturer's ones (Table 1). These results show that the ELISA method used for the three kits was repeatable. 
Table 1. Repeatability of the ELISA method with different kits

\begin{tabular}{|c|c|c|c|c|c|c|c|}
\hline Reagent & $\begin{array}{c}\text { Samples } \\
\text { (Level) }\end{array}$ & $\begin{array}{c}\text { Number of } \\
\text { values (N) }\end{array}$ & Mean & SD & $\begin{array}{c}\text { CV } \\
(\mathbf{\%})\end{array}$ & $\begin{array}{c}\text { Manufactu-rer } \\
\text { CV (\%) }\end{array}$ & Compliant \\
\hline $\begin{array}{c}\text { Bio-Rad } \\
\text { Genscreen }\end{array}$ & Negative & 17 & 0.089 & 0.0047 & $\mathbf{5 . 2 8}$ & $\mathbf{5 . 3 7}$ & Yes \\
$\begin{array}{c}\text { ULTRA- } \\
\text { HIV Ag-Ac }\end{array}$ & Positive & 17 & 2.857 & 0.0768 & $\mathbf{2 . 6 9}$ & $\mathbf{8 . 4 8}$ & Yes \\
$\begin{array}{c}\text { Bio-Rad } \\
\text { Monolisa- } \\
\text { HBs Ag }\end{array}$ & Negative & 17 & 0.0205 & 0.0014 & $\mathbf{6 . 8 3}$ & $\mathbf{1 0 . 6}$ & Yes \\
ULTRA & 17 & 2.5209 & 0.060 & $\mathbf{2 . 3 8}$ & $\mathbf{6 . 2}$ & Yes \\
\hline $\begin{array}{c}\text { Bio-Rad } \\
\text { Monolisa }\end{array}$ & Negative & 17 & 0.069 & 0.0044 & $\mathbf{6 . 3 8}$ & $\mathbf{1 0 . 4}$ & Yes \\
$\begin{array}{c}\text { HCVAg-Ac } \\
\text { ULTRA V2 }\end{array}$ & Positive & 17 & 2.193 & 0.084 & $\mathbf{3 . 8 3}$ & $\mathbf{6 . 1}$ & Yes \\
\hline
\end{tabular}

Table 1 shows the means of optical densities of negative and positive controls of Bio-Rad Genscreen ULTRA-HIV Ag-Ac, Bio-Rad Monolisa-HBs Ag ULTRA and Bio-Rad Monolisa HCV Ag-Ac ULTRA V2 ELISA kits tested 17 times; their standard deviations, their coefficients of variation and the compliance with manufacturer's values.

\section{Evaluation of reproducibility}

The ELISA method reproducibility test with three kits results are presented in Table 2. The BIO-Rad Genscreen ULTRA-HIV Ag-Ac kit negative control $\mathrm{CV}$ was $7.33 \%$ which is lower than manufacturer's $\mathrm{CV}$ (9.9\%). Its positive control CV (5.83\%) was also lower than manufacturer's CV (17.9\%). Similar trends were found for Bio-Rad Monolisa-HBs Ag ULTRA and Bio-Rad Monolisa HCVAg-Ac ULTRA V2 kits controls CV, which were all lower than manufacturer's values (Table 2). These results lead to conclude that ELISA method assayed with the three kits is reproducible.

Table 2. Reproducibility of the ELISA method with different kits

\begin{tabular}{|l|c|c|c|c|c|c|c|}
\hline Reagent & $\begin{array}{c}\text { Samples } \\
\text { (Level) }\end{array}$ & $\begin{array}{c}\text { Number of } \\
\text { values (N) }\end{array}$ & Mean & SD & $\begin{array}{c}\text { CV } \\
\mathbf{( \% )}\end{array}$ & $\begin{array}{c}\text { Manufactu- } \\
\text { rer CV (\%) }\end{array}$ & Compliant \\
\hline $\begin{array}{l}\text { Bio-Rad } \\
\text { Genscreen } \\
\text { ULTRA } \\
\text { HIV Ag-Ac }\end{array}$ & Negative & 17 & 0.086 & 0.0063 & $\mathbf{7 . 3 3}$ & $\mathbf{9 . 9}$ & Yes \\
$\begin{array}{l}\text { Bio-Rad } \\
\text { Monolisa } \\
\text { HBs-Ag } \\
\text { ULTRA }\end{array}$ & Negative & 17 & 0.0198 & 0.0025 & $\mathbf{1 2 . 6 3}$ & $\mathbf{1 8 . 1}$ & Yes \\
\hline
\end{tabular}




\begin{tabular}{|l|c|c|c|c|c|c|c|}
\hline $\begin{array}{l}\text { Bio-Rad } \\
\text { Monolisa }\end{array}$ & Negative & 17 & 0.069 & 0.0083 & $\mathbf{1 2 . 0 3}$ & $\mathbf{1 9 . 4}$ & Yes \\
$\begin{array}{l}\text { HCV Ag- } \\
\text { Ac ULTRA }\end{array}$ & Positive & 17 & 2.476 & 0.298 & $\mathbf{1 2 . 0 4}$ & $\mathbf{1 5}$ & Yes \\
V2 & & & & & & & \\
\hline
\end{tabular}

Table 2 shows the means of optical densities of negative and positive controls of Bio-Rad Genscreen ULTRA-HIV Ag-Ac, Bio-Rad Monolisa-HBs Ag ULTRA and Bio-Rad Monolisa HCV Ag-Ac ULTRA V2 ELISA kits tested 17 times; their standard deviations, their coefficients of variation and the compliance with manufacturer's values.

\section{Sensitivity assessment}

Table 3 shows the results of sensitivity test with different ELISA kits. The sensitivity obtained with Bio-Rad Genscreen ULTRA HIV Ag-Ac kit was $100 \%$ with a positive predictive values (PPV) of 95\%. For Bio-Rad Monolisa HBs Ag ULTRA kit, the sensitivity was equal to the PPV, 98.08\%. Concerning Bio-Rad Monolisa HCV Ag-Ac ULTRA V2 kit, sensitivity was 94.59\% with a PPV of $92.11 \%$ (Table 3). These results were close to manufacturer's values and showed that the ELISA method was sensitive for the three kits.

Table 3. Sensitivity for different ELISA kits

\begin{tabular}{|c|c|c|c|c|c|c|}
\hline Reagent & $\begin{array}{c}\text { Number of } \\
\text { samples }\end{array}$ & FN & FP & $\begin{array}{c}\text { Sensitivity } \\
(\mathbf{\%})\end{array}$ & $\begin{array}{c}\text { PPV } \\
\text { (\%) }\end{array}$ \\
\hline $\begin{array}{c}\text { Bio-Rad } \\
\text { Genscreen } \\
\text { ULTRA HIV } \\
\text { Ag-Ac }\end{array}$ & Manufacturer & 6038 & 0 & 0 & 100 & 100 \\
\hline & Our test & 132 & 0 & 5 & 100 & 95 \\
\hline $\begin{array}{c}\text { Bio-Rad } \\
\text { Monolisa } \\
\text { HBs Ag } \\
\text { ULTRA }\end{array}$ & Manufacturer & 428 & 0 & 0 & 100 & 100 \\
$\begin{array}{c}\text { Bio-Rad } \\
\text { Monolisa }\end{array}$ & Our test & 20 & 1 & 1 & 98.08 & 98.08 \\
$\begin{array}{c}\text { HCV Ag-Ac } \\
\text { ULTRA V2 }\end{array}$ & Our test & 90 & 2 & 3 & 94.59 & 92.11 \\
\hline
\end{tabular}

Table 3 shows the number of samples tested per kit, number of false negative (FN) and false positive (FP) obtained, the sensitivities and the positive predictive values (PPV) for Bio-Rad Genscreen ULTRA-HIV Ag-Ac, 
Bio-Rad Monolisa-HBs Ag ULTRA and Bio-Rad Monolisa HCV Ag-Ac ULTRA V2 ELISA kits.

\section{Specificity assessment}

The results of specificity test with ELISA kits are compiled in Table 4. The specificity value for BIO-Rad Genscreen ULTRA-HIV Ag-Ac kit was $86.49 \%$ with a Negative predictive Values (NPV) of $100 \%$. With BioRad Monolisa-HBs Ag ULTRA, the specificity was equal to the NPV (97.37\%). Bio-Rad Monolisa HCV Ag-Ac ULTRA V2 kit gave a specificity of $94.34 \%$ with a NPV of $96.15 \%$ (Table 4). These results lead to conclude that the ELISA method is more specific for the diagnosis of Hepatitis B and C.

Table 4. Specificity for different ELISA kits

\begin{tabular}{|c|c|c|c|c|c|c|}
\hline Reagent & & $\begin{array}{c}\text { Number of } \\
\text { samples }\end{array}$ & FN & FP & $\begin{array}{c}\text { Specificity } \\
\text { (\%) }\end{array}$ & $\begin{array}{l}\text { NPV } \\
(\%)\end{array}$ \\
\hline $\begin{array}{c}\text { Bio-Rad } \\
\text { Genscreen } \\
\text { ULTRA HIV } \\
\text { Ag-Ac }\end{array}$ & Manufacturer & 6038 & 0 & 0 & 100 & 100 \\
\hline $\begin{array}{c}\text { Bio-Rad } \\
\text { Monolisa } \\
\text { HBs Ag }\end{array}$ & $\begin{array}{c}\text { Manufacturer } \\
\text { Our test }\end{array}$ & 428 & 0 & 0 & $\begin{array}{c}100 \\
97.37\end{array}$ & 100 \\
\hline Bio-Rad & Manufacturer & 501 & 0 & 0 & 100 & 100 \\
\hline $\begin{array}{l}\text { HCV Ag-Ac } \\
\text { ULTRA V2 }\end{array}$ & Our test & 90 & 2 & 3 & 94.34 & 96.15 \\
\hline
\end{tabular}

Table 4 shows the number of samples tested per kit, number of false negative (FN) and false positive (FP) obtained, the specificities and the negative predictive values (NPV) for Bio-Rad Genscreen ULTRA-HIV AgAc, Bio-Rad Monolisa-HBs Ag ULTRA and Bio-Rad Monolisa HCV Ag-Ac ULTRA V2 ELISA kits.

\section{Discussion}

The aim of our study was to evaluate the performance of ELISA method at the serology laboratory of Centre National de Transfusion Sanguine (CNTS) of Lomé, for the diagnosis of HIV, Hepatitis B and C. In fact, HIV, $\mathrm{HBV}$ and $\mathrm{HCV}$ are blood transmittable viruses, so their screening is important for a secure blood transfusion (WHO, 2009). CNTS screen systematically all donors for HBV, HCV, HIV and syphilis. In Togo, Nadjir et al. found among blood donors in CNTS of Lomé, from 2011 to 2015, seroprevalences of 
2.63\%, $1.58 \%$ and $0.92 \%$ for HBV, HCV and HIV, respectively (Koboyo Nadjir et al., 2017).

This study was undertaken in order to correct a discrepancy noted during a diagnostic audit conducted according to the requirements of ISO 15189 standard, in 2016: the non-verification of methods and analytical procedures. The study responds to this challenge by verifying ELISA method used in the serology laboratory of CNTS through reproducibility and repeatability assessments, and also evaluation of sensitivity and specificity. In general, results were compliant with manufacturers one, except HIV diagnosis specificity.

A method evaluation or verification is the confirmation that the recognized method is mastered by the laboratory (SADCAS, 2018). Thus, in China, Li et al. evaluated in 2016, the performance of Elecsys Anti - HCV II assay for HCV screening (Li et al., 2016). Kauffmann-Lacroix et al. in 2013 validated ELISA method for the diagnosis of aspergillosis with BIO-Rad kit (C. Kauffmann-Lacroix , M. Arvier , M. Charron, M.-H. Rodier, 2013). Also, in 2015, Elliot et al. evaluated the diagnostic accuracy of blood centers in the screening of blood donors for viral markers in Ghana (Elliot Eli Dogbe, 2015). In general, the literature on ELISA method verification provides a great deal of information on sensitivity and specificity. In our study, we set out to add repeatability and reproducibility to sensitivity and specificity for a better appreciation.

Repeatability and reproducibility are two important parameters in the assessment of a method precision (International Organization for standardization, 2012).

Repeatability is defined as the variation in measurements performed under the same conditions (Berthold, 2011). It is evaluated by calculating the CV. Here, we found that the CV of negative control (5.28\%) and positive control (2.69\%) with BIO-Rad Genscreen ULTRA-HIV Ag-Ac kit, were lower than the manufacturer's ones, which were $5.37 \%$ and $8.48 \%$ respectively. With Bio-Rad Monolisa-HBs Ag ULTRA kit, the CV of the negative control (6.83\%) and positive control (2.38\%) were also lower than manufacturers' CV (10.6\%; 6.2\%). Same trend was observed with Bio-Rad Monolisa HCV Ag-Ac ULTRA V2 kit negative control CV (6.38\%) and positive control CV (3.83\%) which were lower than manufacturer's CV. These results allowed us to say that ELISA method used at CNTS for the diagnostic of HIV, Hepatitis B and C is repeatable.

On the other hand, Reproducibility is the precision estimate obtained when series of measurements are made under more variable conditions (International Organization for standardization, 2012). We found that BIO-Rad Genscreen ULTRA-HIV Ag-Ac kit negative control CV (7.33\%) was lower than manufacturer's CV (9.9\%). Its positive control CV 
(5.83\%) was also lower than manufacturer's CV (17.9\%). So were Bio-Rad Monolisa-HBs Ag ULTRA and Bio-Rad Monolisa HCV Ag-Ac ULTRA V2 kits controls CV; leading us to conclude that the ELISA method run at CNTS is reproducible.

The accuracy measurements include sensitivity and specificity (Stralen et al., 2009). In our study, sensitivity obtained with Bio-Rad Genscreen ULTRA HIV Ag-Ac kit was 100\%. Hwang et al. found in 2006 a sensitivity of $100 \%$ for Genscreen ULTRA HIV Ag-Ab kit in Korea (Hwang et al., 2006). Abrahim et al. also found in 2019 such a close sensitivity of 99.5\% with the same kit in Ethiopia (Abrahim, 2019), confirming the high sensitivity of the ELISA method used at CNTS for diagnosing HIV.

With the Bio-Rad Monolisa HBs Ag ULTRA kit, we found a sensitivity of $98.08 \%$ whereas Hwang et al. found in Korea in 2006 a sensitivity of $100 \%$ with the same kit (Hwang et al., 2006). Maity et al. also obtained in 2012 a sentivity of 100\% with Microscreen HBsAg ELISA kit in India (Maity et al., 2012). So, the ELISA method for HBV screening at CNTS is sensitive but the little difference between sensitivities, when comparing with authors cited above, could be explained by the lowest number of samples $(\mathrm{N}=20)$ used to perform our sensitivity test.

Regarding the Bio-Rad Monolisa HCV Ag-Ac ULTRA V2 kit, we obtained a sensitivity of 94.59\%. In 2007, Alados-arboledas et al. found a sensitivity of $100 \%$ with Monolisa HCV Ag-Ab ULTRA in Spain (Aladosarboledas et al., 2007). Hwang et al. found the same sensitivity with the same kit in 2006 in Korea (Hwang et al., 2006). This difference must be due to the largest number of samples $(\mathrm{N}>1000)$ used by those authors to perform their test of sensitivity.

In the specificity test, we obtained with BIO-Rad Genscreen ULTRA-HIV Ag-Ac kit a value of $86.49 \%$, which is lower than the one Stanekova et al. found in Slovakia in 2016 (100\%) with Genscreen HIV $1 / 2$ version 2 kit (Stanekova et al., 2016). Maity et al. had also found in India a specificity of 98.9\% with ERBA LISA HIV kit (Maity et al., 2012) in 2012. Thus, the ELISA method used at CNTS for the diagnosis of HIV is of low specificity.

With the Bio-Rad Monolisa-HBs Ag ULTRA kit, the specificity was $97.37 \%$. Indeed, by meta-analysis in 2017 , Amini et al. found such a close pooled specificity of $98.4 \%$ on five studies performed in China, Ghana, Cambodia and Vietnam to evaluate eight enzymatic immune assays for HBs antigen detection (Amini et al., 2017); their results prove that ELISA method for diagnosing HBV at CNTS is specific.

The specificity with the Bio-Rad Monolisa HCV Ag-Ac ULTRA V2 kit was $94.34 \%$ while Lambert et al. had found a specificity of $99.86 \%$ with the same kit in Europe in 2007 (Lambert, 2007). Also, in 2015, Khuroo 
et al. in a meta-analysis found a sensitivity of $99.5 \%$ on thirty studies that had evaluated tests to detect HCV antibodies (Khuroo et al., 2015). So, the ELISA method for the detection of HCV at CNTS was not as specific as others but it can be explained by the difference between kits used in each case.

In this study we deplore that we did only 17 tests instead of 30 as recommended by COFRAC, due to lack of funding. Nevertheless, the results will allow to establish a verification file of the ELISA method used at the serology laboratory of CNTS of Lomé.

\section{Conclusion}

The verification of analytical methods and procedures is a sine qua non step in the quality approach of a medical analysis laboratory. In this study, the verification of the ELISA method used in the CNTS serology laboratory according to the COFRAC SH GTA 04 reference showed that the method is repeatable, reproducible and sensitive for the diagnosis of HIV and hepatitis $\mathrm{B}$ and $\mathrm{C}$. Compared to the diagnostic specificity for hepatitis B and C, the method is less specific for the diagnosis of HIV. Based on these results, this study appears as a contribution to the establishment of a verification file of the ELISA method used at the serology laboratory of CNTS of Lomé.

\section{References:}

1. Abrahim Saro Abdella; Mulu Girma; Abebe Habteselassie; Nigussie Gezahegn; Altaye Feleke; Tezera Moshago Berheto; Minilik Demissie; Wudnesh Belete; Tekalign Deressa. « Diagnostic accuracy of HIV test kits , Genscreen Ultra and Bioelisa ", HIV/AIDS Research and Palliative Care. 2019, vol.11. p. 17-22.

2. Alados-arboledas Juan C; Luis Calbo-torrecillas; M Dolores Lópezprieto et al. « Evaluación de la técnica Monolisa HCV Ag-Ab ULTRA ( BioRad ) en un hospital general ", Enfermedades Infecciosas y Microbiología Clínica. 2007, vol.25 no 3. p. 172-176.

3. Amini Ali; Olivia Varsaneux; Helen Kelly et al. « Diagnostic accuracy of tests to detect hepatitis B surface antigen : a systematic review of the literature and meta-analysis ", BMC Infectious Diseases. 2017, vol.17 no 1. p. 698.

4. Berthold Titertek. «Repeatability and Reproducibility using Crocodile mini Workstation $\gg 2011$ p. 1-6 https://scienze.nz/home/berthold/crocodile-workstations/

5. C. Kauffmann-Lacroix; M. Arvier ; M. Charron; M.-H. Rodier; A. Vassault. " Detection of Aspergillus antigen galactomannan using ELISA method: Validation of the performances of the method for accreditation », J Mycol Med. 2013, vol.23. p. 33-39. 
6. COFRAC. « Exigences pour l'accréditation selon les normes NF EN ISO 15189 et NF EN ISO 22870 ». 2016. 30 p. En ligne : https://tools.cofrac.fr/documentation/SH-REF-02

7. COFRAC. «Guide technique d'accreditation de verification (portee a) / validation (portee b) des methodes en biologie medicale ». 2015. 191 p. https://tools.cofrac.fr/documentation/SH-GTA-04

8. Cucherat, Pierre. «Contribution du pharmacien humanitaire dans une démarche qualité en hygiène hospitalière et biologie médicale au Centre Hospitalier Préfectoral de Kpalimé , région des plateaux , Togo. 2017 ». 165 p. https://dumas.ccsd.cnrs.fr/dumas-01909760

9. Elliot Eli Dogbe, Fareed Arthur. " Diagnostic accuracy of blood centers in the screening of blood donors for viral markers », Pan Afr Med J. 2015, vol.20.p. 1-12.

10. Hwang, Sang-hyun, Heung-bum Oh, Hyon-suk Kim et al. «Evaluation of HBs Ag, HCV and HIV Ag-Ab Assays using Bio-Rad Elite Microplate Analyzer », Korean J Lab Med. 2006, vol.26. p. 436-441.

11. International Organization for standardization. " ISO 15189:2012 Medical laboratories-Requirements for quality and competence ", International Organization for standardization ISO. 2012. https://www.iso.org/standard/56115.html

12. International Standard Organization. " ISO/IEC 17025 General requirements for the competence of testing and calibration laboratories ", International 2005. https://www.iso.org/obp/ui/\#iso:std:iso-iec:17025:ed-2:v1:en

13. ISO 9000. « International Standard ISO 9000:2005 Quality management systems - Fundamentals and vocabulary », International Organization for 2015. https://www.iso.org/obp/ui/\#iso:std:iso:9000:ed-3:v1:en

14. Mehnaaz Sultan Khuroo; Naira Sultan Khuroo; Mohammad Sultan Khuroo. "Diagnostic Accuracy of Point-of-Care Tests for Hepatitis C Virus Infection: A Systematic Review and Meta-Analysis ", PLoS ONE. 2015, vol.10 no 3. p. 1-22.

15. Koboyo Liza Nadjir, Malewe Kolou, Gnatoulma Katawa et al. « Seroprevalence of hepatitis B virus, hepatitis C virus, and human immunodeficiency virus among volunteer blood donors in the National Blood Transfusion Center of Lomé », International Journal of Blood transfusion and Immunohematology. 2017, vol.7. p. 41-45.

16. Lambert, N. « Value of HCV antigen-antibody combined HCV assay in hepatitis C diagnosis », Developments in biologicals. 2007, vol.127. p. 113-121. 
17. Li Dongdong; Siyuan Zhu; Tingting Wang et al. « Comparison of Elecsys Anti-HCV II Assay With Other HCV Screening Assays », Journal of Clinical Laboratory Analysis. 2016, vol.30. p. 451-456.

18. Maity Susmita; Srijita Nandi; Subrata Biswas et al. «Performance and diagnostic usefulness of commercially available enzyme linked immunosorbent assay and rapid kits for detection of HIV , HBV and HCV in India », Virology Journal. 2012, vol.9. p. 290.

19. Marques-Garcia F; Garcia-Codesal MF; Caro-Narros MR; ContrerasSanFeliciano T. " Importance of implementing an analytical quality control system in a core laboratory ", Revista de Calidad Asistencial. 2015, vol.30 no 6 . p. 302-309

20. SADCAS TR 18. "Criteria for validation and quality assurance in microbiological testing ». 2018, no 1. p: 1-37.

21. Stanekova D; Mirandola M; Gios L et al. « Validation study of a conventional enzyme immunoassay to detect HIV antibodies in oral fluid », Bratisl Med J. 2016, vol.117. p. 19-21.

22. Stralen Karlijn J Van; Vianda S Stel; Johannes B Reitsma et al. « Diagnostic methods I: sensitivity, specificity, and other measures of accuracy », International Society of Nephrology. 2009, vol.75. p. 1257-1263.

23. WHO. "Availability , safety and quality of blood products ", WHA63.12 Sixty - Third World Health Assembly. 2010. https://apps.who.int/gb/ebwha/pdf_files/wha63/a63_r12-en.pdf

24. WHO. Screening Donated Blood for Transfusion-Transmissible Infections

\section{Recommendations}

2009. https://www.who.int/bloodsafety/ScreeningTTI.pdf 